\title{
The cost bloodstream infections caused by antimicrobial susceptible and non-susceptible Enterobacteriaceae and Staphylococcus aureus in European hospitals
}

\author{
AJ Stewardson ${ }^{1 *}$, N Graves $^{2}$, A Allignol $^{3}$, S Harbarth ${ }^{1}$, TIMBER Study Group \\ From 3rd International Conference on Prevention and Infection Control (ICPIC 2015) \\ Geneva, Switzerland. 16-19 June 2015
}

\section{Introduction}

Antimicrobial resistance (AMR) represents a significant global threat. It is useful to estimate the economic burden of AMR as these represent potential cost savings from reducing the problem.

\section{Objectives}

To determine the impact of antimicrobial resistance on cost attributable to bloodstream infections (BSIs) caused by Staphylococcus aureus and Enterobacteriaceae from a European hospital perspective.

\section{Methods}

We performed a multicentre retrospective cohort study including acute inpatient episodes at ten European hospitals in 2010 and 2011. BSIs were the time-varying exposure of interest, with $S$. aureus classified as methicillin-susceptible (MSSA) or resistant (MRSA), and Enterobacteriaceae as third-generation-cephalosporin-susceptible (3GCSE) or resistant (3GCRE). We used multistate models to estimate excess length-of-stay (LOS). For each bacteria/susceptibility combination, we computed the attributable cost of a single BSI as the product of excess LOS and the bed-day value. We used two contrasting bed-day values: an economic value obtained by contingent valuation and the accounting cost derived by dividing the annual hospital budget by the number of bed-days. To estimate the annual hospital costs of each BSI, these BSI costs were multiplied by the expected number of BSI cases per year. We performed a probabilistic sensitivity analysis to account for parameter uncertainty.

University of Geneva Hospitals, Geneva, Switzerland

Full list of author information is available at the end of the article

\section{Results}

Our cohort included 606,649 patients. Third-generationcephalosporin-resistance significantly increased the hazard of death (1.5 [1.0-2.2]), excess LOS (4.9 [1.1-8.7]) and cost compared to susceptible strains, whereas methicillin resistance did not. Whilst 3GCSE BSI was associated with the lowest per-infection cost $(€ 320$ [95\% credible interval, €80-€1,300] or $€ 4,000[€ 2,400-€ 6,700]$ using economic and accounting valuations, respectively), their relative frequency resulted in equal highest annual cost with MSSA $(€ 77,000[€ 19,000-€ 300,000]$ or $€ 970,000[€ 590,000-€ 1,600,000]$ using economic and accounting valuations, respectively).

\section{Conclusion}

While BSI with S. aureus has a greater impact on mortality, excess LOS and cost than Enterobacteriaceae per infection, the impact of antimicrobial resistance is greater amongst BSIs caused by Enterobacteriaceae.

\section{Disclosure of interest}

None declared.

\section{Authors' details}

${ }^{1}$ University of Geneva Hospitals, Geneva, Switzerland. ${ }^{2}$ Queensland University of Technology, Brisbane, Queensland, Australia. ${ }^{3}$ Ulm University, Ulm, Germany.

Published: 16 June 2015

doi:10.1186/2047-2994-4-S1-055

Cite this article as: Stewardson et al:: The cost bloodstream infections caused by antimicrobial susceptible and non-susceptible

Enterobacteriaceae and Staphylococcus aureus in European hospitals. Antimicrobial Resistance and Infection Control 2015 4(Suppl 1):055. 